\title{
(2) OPEN ACCESS \\ Circulating lipid and lipoprotein profiles and their correlation to cardiac function and cardiovascular outcomes in patients with acute myocardial infarction
}

\author{
Haoyu Wu, ${ }^{1}$ Chen Wang, ${ }^{1}$ Gulinigaer Tuerhongjiang, ${ }^{1}$ Xiangrui Qiao, ${ }^{1}$ \\ Yiming Hua, ${ }^{1}$ Jianqing She (1) , 1,2,3 Zuyi Yuan ${ }^{1,2,3}$
}

\begin{abstract}
${ }^{1}$ Department of Cardiovascular Medicine, Xi'an Jiaotong University Medical College First Affiliated Hospital, Xi'an, China

${ }^{2}$ Key Laboratory of Molecular Cardiology, Xi'an Jiaotong University, Xi'an, China ${ }^{3}$ Key Laboratory of Environment and Genes Related to Diseases, Xi'an Jiaotong University Ministry of Education, Xi'an, China
\end{abstract}

\section{Correspondence to Professor Zuyi Yuan, Department of Cardiovascular Medicine, Xi'an Jiaotong University Medical College First Affiliated Hospital, Xi'an, Shaanxi 710061, China; zuyiyuan@mail.xjtu.edu.cn Dr Jianqing She; jianqingshe@xjtu.edu.cn}

HW and CW contributed equally.

Accepted 13 April 2021 Published Online First 10 May 2021

\section{Check for updates}

(C) American Federation for Medical Research 2021. Re-use permitted under CC BY-NC. No commercial re-use. Published by BMJ.

To cite: Wu H, Wang $C$, Tuerhongjiang $\mathrm{G}$,

et al. J Investig Med

2021;69:1310-1317.

\section{ABSTRACT}

Recent studies showed that lipoproteins represent major risk factors, both positive and negative, for atherosclerotic cardiovascular disease. The aim of the present study was to describe the relationship between plasma lipid profile and cardiac function and cardiovascular outcomes in patients with acute myocardial infarction (AMI) after percutaneous coronary intervention (PCI). Two independent groups of subjects including a total of 797 patients diagnosed of AMI undergoing $\mathrm{PCl}$ admitted to the First Affiliated Hospital of Xi'an Jiaotong University were included in the present study. We performed a cross-sectional study for the correlation between plasma lipid profile and cardiac function based on the first group, including 503 patients with AMI. We further validated the correlation and did the followup of 2.4 years of major cardiovascular outcomes on the second group, including 294 patients with AMI. Our results showed that apolipoprotein A-I (ApoA-I) level was significantly reduced, and the high-density lipoprotein cholesterol (HDL-C):ApoA-I ratio was increased in the patients with lower LVEF or higher $\mathrm{N}$-terminal pro-B-type natriuretic peptide levels compared with the control; there was a positive correlation between cardiac function and ApoA-I, and a negative correlation between cardiac function and the HDL-C:ApoA-I ratio. Meanwhile, multivariate Cox analysis showed that ApoA-I was independent predictors of major adverse cardiovascular events (MACEs). Kaplan-Meier survival analysis showed the ApoA-I levels exhibited a significant effect on predicting the incidence of MACEs. In sum, plasma ApoA-I level is positively associated with the cardiac function of patients with $\mathrm{AMI}$ after $\mathrm{PCl}$, and $\mathrm{ApoA}-\mathrm{I}$ is an independent indicator to predict the incidence of MACEs.

\section{INTRODUCTION}

Coronary artery disease (CAD) is the major cause of mortality and morbidity in China and worldwide. Despite the technological advancement and the increasing level of awareness, ${ }^{12}$ acute myocardial infarction (AMI) is still a lifethreatening emergency, and long-term chronic

\section{Significance of this study}

What is already known about this subject?

- The attack of acute myocardial infarction (AMI) is life-threatening, and long-term chronic ischemia of the myocardium will cause adverse clinical outcomes, such as ischemic heart failure as well as fatal arrythmia.

- The abnormal lipid metabolism took a critical part in plaque formation and atherosclerosis in the development of AMI.

- However, few studies were concerned about the relationship between whole lipid types and cardiac function, and the correlation of prognosis with lipid profile remains controversial due to the lack of long-term follow-up results.

What are the new findings?

- In this study, we found that apolipoprotein A-I (ApoA-I) levels were significantly reduced, and the high-density lipoprotein cholesterol (HDL-C):apolipoprotein A-I (ApoA-I) ratio were increased in the patients with lower LVEF or higher N-terminal pro-B-type natriuretic peptide level.

- The Pearson correlation analysis showed positive correlations between cardiac function and ApoA-I, and negative correlations between cardiac function and the HDL-C:ApoA-I ratio.

- Moreover, ApoA-I levels exhibited a significant effect on predicting the incidence of major adverse cardiovascular events (MACEs).

How might these results change the focus of research or clinical practice?

- The present study provides broad and straightforward support that ApoA-I should be introduced into clinical practice for the assessment of the cardiac function in patients with AMI undergoing percutaneous coronary intervention, and also predicts the incidence of MACEs. 
ischemia of the myocardium will cause adverse clinical outcomes, such as ischemic heart failure as well as fatal arrythmia. ${ }^{3}$ Actually, the condition of patients with AMI who may have poor prognosis could be greatly improved by timely and appropriate interventions. Based on this, finding an efficient predictor related with cardiac function and cardiovascular outcomes is urgently needed.

Lipid abnormalities have been widely documented to be associated with higher cardiovascular disease (CVD) risk. ${ }^{4}$ Widely used clinical CVD risk calculators frequently include classical biochemistry measures of total cholesterol (TC), low-density lipoprotein cholesterol (LDL-C), or a combination of these. ${ }^{5-7}$ However, the predictive value of non-traditional lipid risk factors has also gained increasing attention from researchers, including high-density lipoprotein cholesterol (HDL-C), non-HDL, apolipoprotein A-I (ApoA-I), etc. ${ }^{8}$ HDL-C was ascribed as 'good' cholesterol and negatively correlates to the risk of CVDs as proven by several clinical and animal studies. ${ }^{9-12}$ Non-HDL-C has been suggested as a pragmatic and cost-effective alternative to direct LDL-C measurement, also proven to be associated with increased CVD risk. ${ }^{13}$ ApoA-I is the principal protein component of HDL particles and is also of interest for its potential value for predicting CVD risks. ${ }^{14-16}$ Specifically, ApoA-I level is a consistent discriminator of atherosclerotic burden among patients with stable CAD ${ }^{17}$ However, the correlation between ApoA-I and cardiac function, as well as long-term outcomes in patients with AMI undergoing percutaneous coronary intervention (PCI), remain underexplored.

With these considerations, our work was conducted to evaluate the lipid profile of patients with AMI after PCI and the relationship among dyslipidemia, cardiac function and long-term cardiovascular outcomes.

\section{MATERIALS AND METHODS Study population}

This study enrolled two independent groups of subjects including a total of 797 patients diagnosed with AMI undergoing PCI, admitted to the First Affiliated Hospital of $\mathrm{Xi}$ 'an Jiaotong University. We performed a cross-sectional study of the correlation between plasma lipid profile and cardiac function based on the first group including 503 patients with AMI admitted between January 2013 and December 2015. We further validated the correlation and did the follow-up of 2.4 years on the second group including 294 patients with AMI between January 2016 and December 2016; 28 (9.52\%) patients were lost follow-up. AMI was defined based on the universal definition criteria by the joint European Society of Cardiology (ESC)/American College of Cardiology Foundation/American Heart Association/World Heart Federation Task Force. ${ }^{18}$ The exclusion criteria were (1) age $<18$ years, (2) pregnancy, (3) renal dysfunction (serum creatinine $>133 \mu \mathrm{mol} / \mathrm{L}$ ) or liver dysfunction (serum alanine transaminase $>3$ times the upper normal limit), and (4) malignant tumors. All patients received guideline-recommended therapy for AMI. The detailed demographic, clinical, drug, hematological, echocardiography and angiographic data were obtained from the hospital documentation.
The estimation of sample size was performed using $G^{*}$ Power software V.3.1.9.6. ${ }^{19}$ A sample size of 252 achieves $95 \%$ power to detect an effect size of 0.25 using $\mathrm{F}$ tests with a significance level of 0.05 .

\section{Lipid profile measures}

Venous blood samples for lipidomic analyses were collected before coronary catheterization. The following laboratory assays were performed in the clinical laboratory department: TC and triglyceride (TG) were detected using detection kit from FUJIFILM via N-(3-sulfopropyl)3-methoxy-5-methylaniline (HMMPS) method; HDL-C and LDL-C were detected using detection kit via direct measurement method from FUJIFILM; ApoA-I, Apo B and Apo E were measured using a detection kit from SEKISUI by turbidimetric inhibition immunoassay. All laboratory assays were performed in duplicate and the results were averaged.

\section{Other blood biochemical measures}

Standard clinical biochemical and hematological measures were made by the local laboratory of the First Affiliated Hospital of Xi'an Jiaotong University. Serum was collected for analysis including liver, kidney and electrolytes (HITACHI 7180; HITACHI, Tokyo, Japan). Full blood samples were used to test the hematological parameters (KX $21 \mathrm{n}$ analyzers; Sysmex, Kobe, Japan). After these tests, all samples were stored at $-80^{\circ} \mathrm{C}$ for future analysis. The serum N-terminal pro-B-type natriuretic peptide (NT-proBNP) levels were detected as a batch analysis in a central laboratory by electrochemiluminescence immunoassay (Roche Diagnostics, Rotkreuz, Switzerland).

\section{Evaluation of the echocardiography}

Echocardiography was performed using Philips iE33 ultrasound system (Philips, Amsterdam, Netherlands) by experienced cardiologists of the First Affiliated Hospital of Xi'an Jiaotong University. The left ventricular ejection fraction (LVEF) value was uniformly measured by biplane Simpson rule. $^{20}$

\section{Assessment of outcomes}

Patients in the follow-up cohort were followed up semiannually by clinic visits or by telephone interviews conducted by trained nurses or doctors. Major adverse cardiovascular event (MACE) is defined as the end point of this study, which referred to the composite of all-cause death, heart failure, non-fatal myocardial infarction (MI), and symptomdriven revascularization. The follow-up ended on June 31, 2018 or patient death.

\section{Statistical analysis}

All statistical analyses were performed by SPSS V.22.0 for Windows. Data were presented as frequencies and percentages for categorical variables, as mean \pm SD for normally distributed continuous variables and median (with 25th and 75 th percentiles) for non-normally distributed continuous variables. The Kolmogorov-Smirnov test was used to assess normal distribution of quantitative variables. Simple t-test was used to compare continuous variables which are in normal distribution. Kruskal-Wallis test was used to compare continuous variables which do not conform 


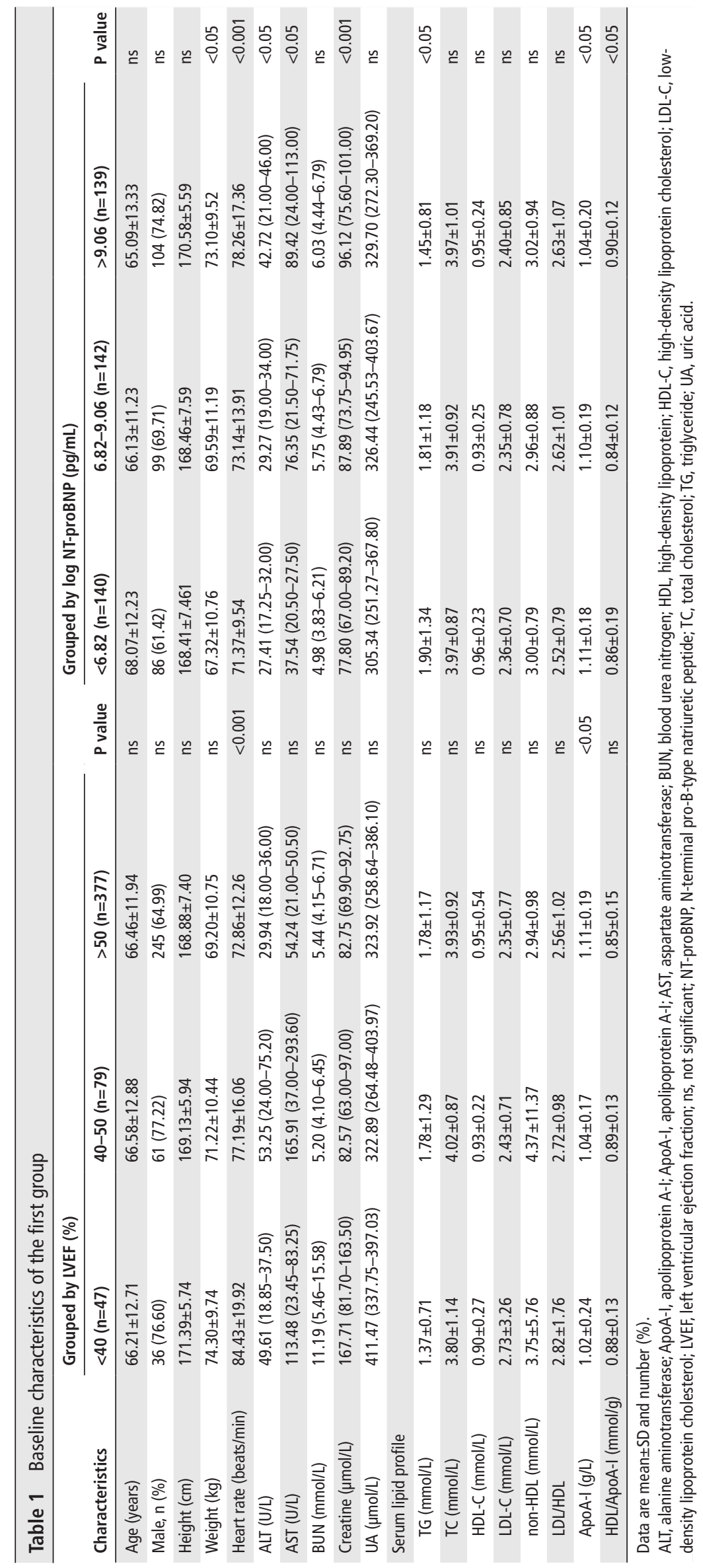


to the normal distribution. $\chi^{2}$ test was used to compare categorical variables. To describe the relationship between plasma lipid profile and cardiac function, patients were divided into subgroups according to the baseline LVEF levels and the NT-proBNP level. The cut-off point of LVEF was defined based on the 2016 ESC guidelines for the diagnosis and treatment of acute and chronic heart failures, which represent varying degrees of cardiac function $(<40 \%, 40 \%-50 \%$, and $>50 \%)$. To limit the influence of extreme observations, NT-proBNP was natural logarithmically transformed to obtain log NT-proBNP. Then patients were grouped according to the tertiles of baseline $\log$ NT-proBNP level. Simple linear analysis was used to calculate the correlation between plasma lipid profile and cardiac function. Univariate and multivariate survival analyses involving Cox regression analysis were constructed to calculate HRs and 95\% CIs for MACEs. The multivariable Cox models were adjusted by age, sex, height, weight, creatinine, LVEF, and NT-proBNP. To assess the prognostic value of the ApoA-I level, Kaplan-Meier survival curves were used and compared by log-rank test. All probability values were two-tailed. A p value of $<0.05$ was considered statistically significant.

\section{RESULTS}

\section{Baseline characteristics of the first group}

A total of 503 patients with a diagnosis of AMI after PCI were enrolled in the first group. The patients were grouped according to the baseline LVEF levels ( $<40 \%, 40 \%-50 \%$, and $>50 \%)$. Baseline characteristics of patients in different LVEF subgroups are shown in table 1. Compared with the patients in the group with higher levels of LVEF, the patients with lower levels of LVEF showed a higher level of heart rate $(84.43 \pm 19.92$ vs $72.86 \pm 12.26, \mathrm{p}<0.001)$. In addition, the patients with lower levels of LVEF had significantly lower levels of ApoA-I level $(1.02 \pm 0.24$ vs $1.11 \pm 0.19, \mathrm{p}<0.05)$ in the plasma. Furthermore, no differences were found in other lipids levels among different LVEF groups, such as TG, TC, LDL-C, HDL-C, non-HDL, the HDL-C:LDL- $\mathrm{C}$ ratio and the HDL-C:ApoA-I ratio. No differences in other risk factors were found between different LVEF level groups, such as age, gender, alanine aminotransferase (ALT), aspartate aminotransferase (AST), creatine, and blood urea nitrogen (BUN).
As shown in table 1, the patients were grouped according to the baseline NT-proBNP level, which was transformed by natural logarithm $(<6.82,6.82-9.06$, and $>9.06)$. We found that the patients with higher levels of log NT-proBNP also had significantly higher levels of weight, heart rate, ALT, AST, creatine and the HDL-C:LDL- $\mathrm{C}$ ratio but lower TG and ApoA-I level in the plasma.

\section{Correlation between lipid and lipoprotein profiles and cardiac function in patients with AMI in the first group} As shown in table 2, Pearson correlation analysis also showed a significantly positive correlation between ApoA-I level and LVEF $(r=0.165, p<0.001)$ but a significantly negative correlation between plasma ApoA-I level and $\log$ NT-proBNP $(r=-0.181, \mathrm{p}<0.001)$. Interestingly, the HDL-C:ApoA-I ratio was positively correlated with the $\log$ NT-proBNP level $(r=0.14, p<0.05)$, and the TG level was negatively correlated with the log NT-proBNP level $(\mathrm{r}=-0.171, \mathrm{p}<0.05)$.

\section{Baseline characteristics of the second group}

A total of 294 patients with a diagnosis of AMI after PCI were enrolled in the validation cohort. The patients were also grouped according to the baseline LVEF level $(<40 \%$, $40 \%-50 \%$, and $>50 \%)$ and the log NT-proBNP level $(<7.51$, $7.51-9.58$, and $>9.58$ ). Compared with patients in the group with the highest level of LVEF, the patients in the group with lower levels of LVEF had a higher level of heart rate $(80.13 \pm 17.96$ vs $69.05 \pm 11.45, \mathrm{p}<0.001)$ and lower levels of ApoA-I level $(1.00 \pm 0.23$ vs $1.08 \pm 0.18, \mathrm{p}=0.175)$ (table 3 ). In addition, the patients with lower levels of LVEF had significantly higher levels of AST, BUN, creatine and UA. We found the same results in the different log NT-proBNP level group analysis. Interestingly, the patients with higher levels of log NT-proBNP also had significantly lower levels of ApoA-I but higher levels of HDL-C:ApoA-I ratio (table 3).

\section{Correlation between plasma lipid profile and cardiac function in patients with AMI in the second group}

As shown in table 4, Pearson correlation analysis also showed a significantly positive correlation between ApoA-I level and LVEF $(r=0.165, \mathrm{p}<0.05)$ but a significantly negative correlation between ApoA-I level and log NT-proBNP $(r=-0.23, p<0.001)$. Interestingly, the HDL-C:ApoA-I

Table 2 Correlation between serum lipid profile and cardiac function in patients with acute myocardial infarction in the first group

\begin{tabular}{|c|c|c|c|c|c|c|}
\hline \multirow[b]{2}{*}{ Characteristics } & \multicolumn{3}{|l|}{ LVEF } & \multicolumn{3}{|l|}{ Log NT-proBNP } \\
\hline & Pearson correlation & Sig. (two-tailed) & $\mathrm{N}$ & Pearson correlation & Sig. (two-tailed) & $\mathbf{N}$ \\
\hline TG (mmol/L) & 0.088 & ns & 471 & -0.171 & $<0.05$ & 402 \\
\hline $\mathrm{TC}(\mathrm{mmol} / \mathrm{L})$ & -0.023 & ns & 469 & -0.02 & ns & 399 \\
\hline $\mathrm{HDL}-\mathrm{C}(\mathrm{mmol} / \mathrm{L})$ & 0.081 & ns & 470 & -0.037 & ns & 401 \\
\hline LDL-C (mmol/L) & -0.031 & ns & 470 & 0.008 & ns & 401 \\
\hline Non-HDL (mmol/L) & -0.051 & ns & 467 & -0.009 & ns & 398 \\
\hline LDL/HDL & -0.07 & ns & 470 & 0.04 & ns & 401 \\
\hline ApoA-I (g/L) & 0.165 & $<0.001$ & 469 & -0.181 & $<0.001$ & 400 \\
\hline HDL-C/ApoA-I (mmol/g) & 0.039 & ns & 471 & 0.14 & $<0.05$ & 400 \\
\hline
\end{tabular}

ApoA-I, apolipoprotein A-I; ApoA-I, apolipoprotein A-I; HDL-C, high-density lipoprotein cholesterol; LDL-C, low-density lipoprotein cholesterol; LVEF, left ventricular ejection fraction; ns, not significant; NT-proBNP, N-terminal pro-B-type natriuretic peptide; TC, total cholesterol; TG, triglyceride. 


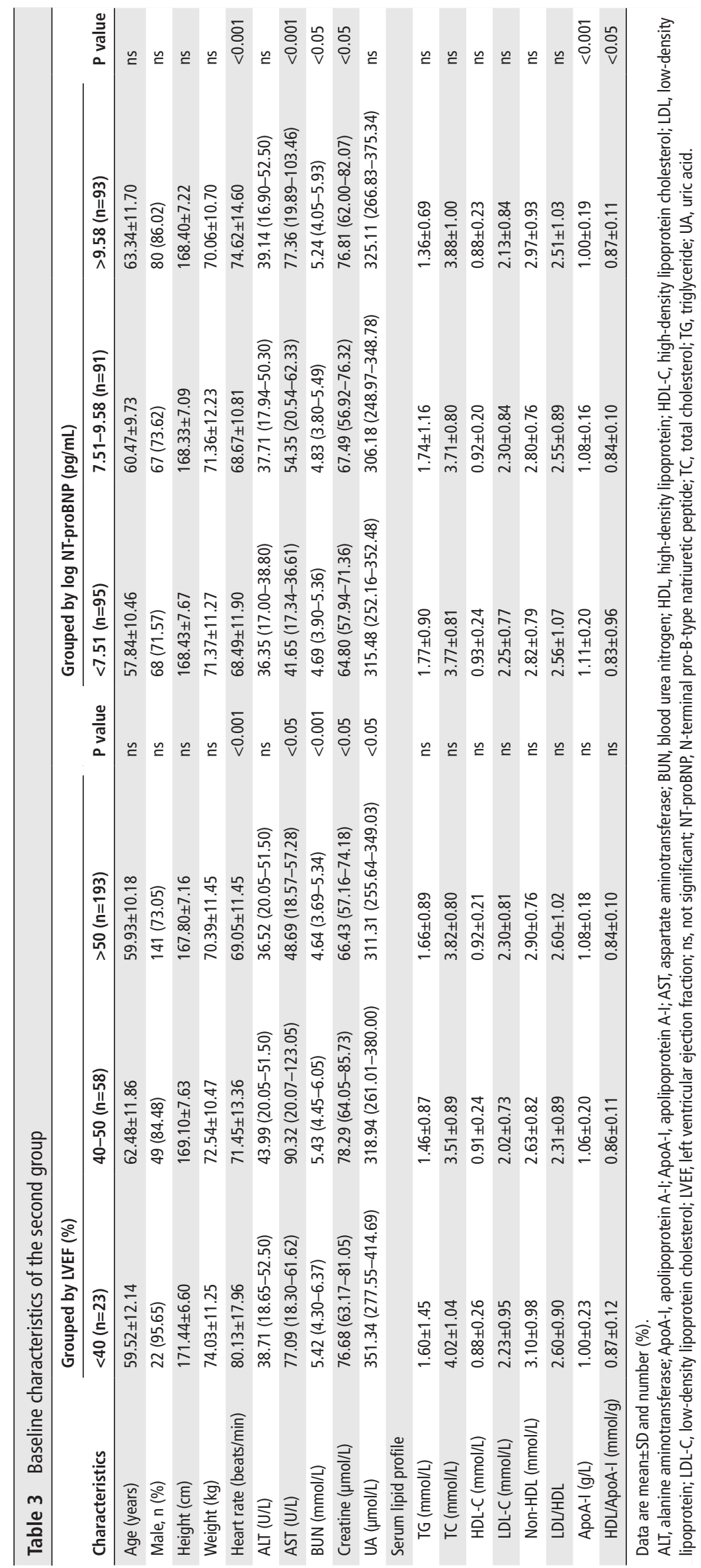


Table 4 Correlation between serum lipid profile and cardiac function in patients with acute myocardial infarction in the second group

\begin{tabular}{|c|c|c|c|c|c|c|}
\hline \multirow[b]{2}{*}{ Characteristics } & \multicolumn{3}{|l|}{ LVEF } & \multicolumn{3}{|l|}{ Log NT-proBNP } \\
\hline & Pearson correlation & Sig. (two-tailed) & N & Pearson correlation & Sig. (two-tailed) & $\mathrm{N}$ \\
\hline TG (mmol/L) & 0.035 & ns & 271 & -0.175 & $<0.001$ & 277 \\
\hline $\mathrm{TC}(\mathrm{mmol} / \mathrm{L})$ & 0.093 & ns & 169 & -0.01 & ns & 165 \\
\hline $\mathrm{HDL}-\mathrm{C}(\mathrm{mmol} / \mathrm{L})$ & 0.111 & ns & 271 & -0.085 & ns & 277 \\
\hline LDL-C (mmol/L) & 0.100 & ns & 271 & -0.08 & ns & 277 \\
\hline Non-HDL (mmol/L) & 0.076 & ns & 169 & 0.01 & ns & 165 \\
\hline LDL/HDL & 0.036 & ns & 271 & -0.03 & ns & 277 \\
\hline ApoA-I (g/L) & 0.165 & $<0.05$ & 271 & -0.23 & $<0.001$ & 277 \\
\hline HDL-C/ApoA-I (mmol/g) & -0.028 & ns & 271 & 0.181 & $<0.05$ & 277 \\
\hline
\end{tabular}

ApoA-I, apolipoprotein A-I; ApoA-I, apolipoprotein A-l; HDL, high-density lipoprotein; HDL-C, high-density lipoprotein cholesterol; LDL, low-density lipoprotein; LDL-C, low-density lipoprotein cholesterol; LVEF, left ventricular ejection fraction; ns, not significant; NT-proBNP, N-terminal pro-B-type natriuretic peptide; TC, total cholesterol; TG, triglyceride.

ratio was positively correlated to the $\log$ NT-proBNP level $(r=0.14, p<0.05)$, and the TG level was negatively correlated to the log NT-proBNP level $(\mathrm{r}=-0.175, \mathrm{p}<0.05)$.

\section{ApoA-I level as an independent predictor of MACE occurrence}

During the median of 28.57 months of follow-up period, $76(25.90 \%)$ patients experienced MACEs. Kaplan-Meier curves were used to illustrate the survival free from adverse events in different ApoA-I level groups in patients with AMI undergoing PCI, as shown in figure 1 . Overall, patients with lower ApoA-I levels had a significantly worse outcome of survival free from MACEs during the follow-up period. Kaplan-Meier survival analysis demonstrated that lower admission ApoA-I level was significantly associated with MACE occurrence $(p<0.001, \log$-rank test).

We then used Cox regression model for further analysis as shown in table 5. In univariate Cox analysis, we found that the lower ApoA-I level was significantly associated with an increased risk of MACEs in patients with AMI undergoing PCI (HR 2.294, 95\% CI 1.239 to 4.248; $\mathrm{p}=0.008$ ) over a median of 2.4 years of follow-up. This relationship

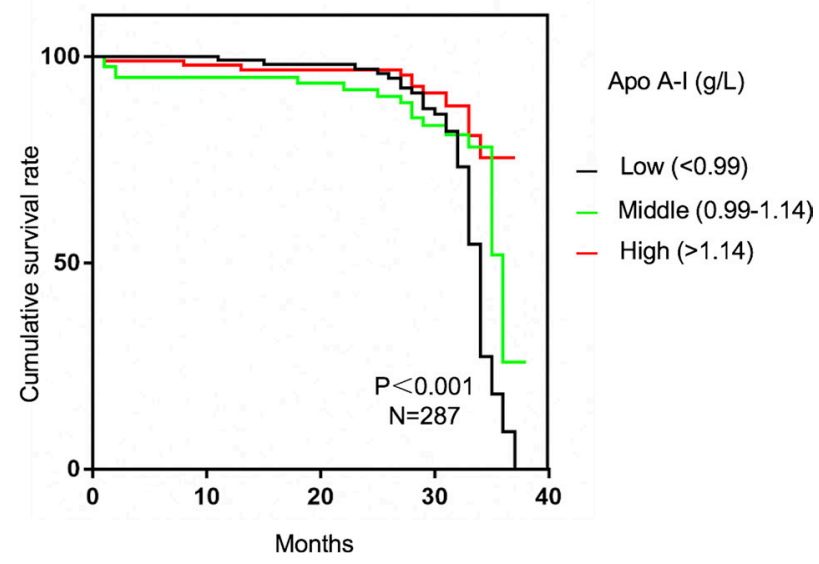

Figure 1 Kaplan-Meier analysis of MACEs based on the ApoA-I levels. The 294 patients were divided by tertiles of the ApoA-I levels: $0.99,0.99-1.14$, and $>1.14 \mathrm{~g} / \mathrm{L}$. Risk of a MACE increased with decreasing tertile of the ApoA-I levels (log-rank test 33.354, $\mathrm{p}<0.001$ ). ApoA-I, apolipoprotein A-I; MACE, major adverse cardiovascular event. remained significant in multivariate Cox analysis (HR 3.411, 95\% CI 1.373 to $8.665 ; \mathrm{p}=0.008$ ) after adjustment for age, sex, height, weight, creatinine, LVEF, and NT-proBNP.

\section{DISCUSSION}

CVD, especially AMI, is still the leading cause of death in China and worldwide, and its morbidity and mortality have continued to increase in recent years. ${ }^{1} 2$ Despite advances in therapeutic strategies for AMI, patients remain at a high risk of MACEs, particularly in the immediate weeks to months after the event. ${ }^{21}$ Dyslipoproteinemia is common in patients with AMI and usually predicts recurrent cardiovascular events. ${ }^{22} 23$ In the present study, we assessed the relationship between circulating lipid and lipoprotein profiles to the cardiac function and cardiology outcomes in patients with AMI undergoing PCI. Our results showed that ApoA-I levels were significantly reduced, and the HDL-C:ApoA-I ratios were increased in the patients with lower LVEF or higher NT-proBNP levels; there were positive correlations between cardiac function and ApoA-I, and negative correlations between cardiac function and the HDL-C:ApoA-I ratio. The ApoA-I levels exhibited a significant effect on predicting the incidence of MACEs.

The major novelty is that in the present study, we have demonstrated the utility of ApoA-I for predicting future adverse cardiovascular events in patients with AMI undergoing PCI from two clinical groups. Findings from the Incremental Decrease in End Points Through Aggressive Lipid Lowering (IDEAL) study concur that levels of ApoA-I were higher compared with their CAD population without heart failure among patients with new-onset heart failure. ${ }^{24} \mathrm{~A}$ large cohort of Controlled Rosuvastatin Multinational Trial in Heart Failure (CORONA) study proved that higher baseline HDL and ApoA-I were associated with a better prognosis; particularly, ApoA-I was more predictive than LDL or HDL. ${ }^{25}$ Moreover, a cross-sectional study of 199 patients with stable CAD also found that ApoA-I levels increased with increasing NYHA class. ${ }^{24}$ In our study, we have identified the correlation between ApoA-I and the cardiac function of patients with AMI after PCI. The cardiac function is strongly associated with the changes in lipid profile, with positive correlations between cardiac function and ApoA-I, and negative correlations between cardiac function and the HDL-C:ApoA-I ratio. 
Table 5 Univariate and multivariate Cox analysis for MACEs in the second group

\begin{tabular}{|c|c|c|c|c|c|c|}
\hline \multirow[b]{2}{*}{ Variable } & \multicolumn{3}{|c|}{ Univariate analysis } & \multicolumn{3}{|c|}{ Multivariate analysis } \\
\hline & HR & $95 \% \mathrm{Cl}$ & $P$ value & HR & $95 \% \mathrm{Cl}$ & $P$ value \\
\hline \multicolumn{7}{|l|}{ ApoA-I levels } \\
\hline High $(>1.14 \mathrm{~g} / \mathrm{L})$ & Reference & & & Reference & & \\
\hline Middle (0.99-1.14 g/L) & 1.484 & 0.713 to 3.091 & 0.291 & 1.734 & 0.649 to 4.631 & 0.272 \\
\hline Low $(<0.99 \mathrm{~g} / \mathrm{L})$ & 2.294 & 1.239 to 4.248 & 0.008 & 3.411 & 1.373 to 8.665 & 0.008 \\
\hline
\end{tabular}

Adjusted for age, sex, height, weight, creatinine, left ventricular ejection fraction, and N-terminal pro-B-type natriuretic peptide.

ApoA-I, apolipoprotein A-I; $95 \%$ Cl, 95 \% confidence interval; HR, hazard ratio; MACE, major adverse cardiovascular event.

Furthermore, ApoA-I is an independent indicator to predict the incidence of MACEs. It is identified that ApoA-I is a key functional apolipoprotein component of $\mathrm{HDL}$ particles and plays a central role in cholesterol efflux, and has also been of interest for predicting CVD risks. ${ }^{26}{ }^{27}$ In agreement with our results, the Apo-I Event Reducing in Ischemic Syndromes-I (AEGIS-I) trial, a phase IIb trial of patients with recent MI, found that a reconstituted ApoA-I (CSL112) was developed to enhance cholesterol efflux capacity. Notably, CSL112 was safe and confirmed its potential to remove cholesterol from atherosclerotic plaques. ${ }^{28-30}$ A meta-analysis proved that incident CVD events occurred more frequently in those subjects with lower ApoA-I, and ApoA-I had the strongest (inverse) associations with risk of fatal CVD. ${ }^{31} 32$ A case-control study found that ApoA-I was inversely related to mortality: for each $1 \mathrm{SD}$ increase of ApoA-I, 31\% and 33\% decreases in all-cause and cardiovascular mortality were recorded. ${ }^{33}$ So far, ApoA-I has been little used in epidemiological studies. Furthermore, ApoA-I measurement is much less influenced than HDL-C by intravascular enzymes and lipid transfer proteins, which participate in HDL remodeling. Thus, ApoA-I measurement may improve assessment of cardiovascular risk. ${ }^{34}$

In this study, we evaluated the circulating levels of TG, TC, HDL, LDL, non-HDL, ApoA-I, etc. It is interesting that only ApoA-I showed its correlation to cardiac function. Theoretically, there are several reasons. First, HDL protects against atherosclerosis through multiple mechanisms, including amelioration of endothelial dysfunction, removal of excess cholesterol from macrophages, as well as antioxidative, anti-inflammatory, and antiapoptotic effects. ApoA-I is the primary functional apolipoprotein component of HDL, which plays pivotal roles in the reverse cholesterol transport pathways by modulating HDL-C formation, stabilization, binding to the hepatic scavenger receptors, and activating lecithin cholesterol acyl transferase. Therefore, the oxidation of particular residues on ApoA-I creates a dysfunctional HDL particle that is associated with an increased incidence of cardiovascular events. ${ }^{9} 103536$ Our data provide evidence that ApoA-I could be introduced into clinical practice for assessing the cardiac function of patients with AMI undergoing PCI and for predicting the incidence of MACEs.

\section{LIMITATIONS}

The present study had several limitations. First, this was a single-center study restricted to Chinese patients with AMI after PCI. As mentioned previously, caution should be exercised when generalizing our findings to other ethnic groups, and further studies involving different ethnic groups are needed to support our findings. Moreover, we did not collect any data whether the patients had taken any medication (particularly lipid-lowering medication) during the 2.4 years of follow-up and, if they were (which is most likely), which medication and in what doses.

\section{CONCLUSION}

In summary, our results demonstrate that ApoA-I levels were significantly reduced, and the HDL-C:ApoA-I ratios were increased in the patients with lower LVEF or higher NT-proBNP level compared with the control. Pearson correlation analysis further showed positive correlations between cardiac function and ApoA-I and negative correlations between cardiac function and the HDLC:ApoA-I ratio. In addition, the ApoA-I levels exhibited a significant effect on predicting the incidence of MACEs. Therefore, the ApoA-I level was positively associated with the cardiac function of patients with AMI after PCI, and ApoA-I is an independent indicator to predict the incidence of MACEs.

Contributors JS and ZY designed the study. HW, CW, SQ, YH and JS collected the data. HW and CW analyzed the data. JS and HW wrote the paper.

Funding This study was supported by National Natural Science Foundation of China (81500219, 81400302, and 81570406), the Natural Science Foundation of Shaanxi province (2018KW067, 2017JM8016, and 2016SF217), and the Fundamental Research Funds for the Central Universities in China (1191329724 and 191329849), the Clinical Research Award of the First Affiliated Hospital of Xi'an Jiaotong University, China (No. XJTU1AFCRF-2018-025).

\section{Competing interests None declared.}

Patient consent for publication Not required.

Ethics approval Ethical approval was obtained from the ethics committee of the First Affiliated hospital of Xi'an Jiaotong University. Written informed consent was obtained from all study subjects. All steps of the study conformed to the Helsinki Declaration.

Provenance and peer review Not commissioned; externally peer reviewed.

Data availability statement The datasets used and/or analyzed during the present study are available from the corresponding author on reasonable request.

Open access This is an open access article distributed in accordance with the Creative Commons Attribution Non Commercial (CC BY-NC 4.0) license, which permits others to distribute, remix, adapt, build upon this work noncommercially, and license their derivative works on different terms, provided the original work is properly cited, an indication of whether changes were made, and the use is non-commercial. See: http://creativecommons.org/ licenses/by-nc/4.0/.

\section{ORCID iD}

Jianqing She http://orcid.org/0000-0001-7030-2889 


\section{REFERENCES}

1 Virani SS, Alonso A, Benjamin EJ, et al. Heart disease and stroke statistics-2020 update: a report from the American Heart Association. Circulation 2020;141:e139-596.

2 Timmis A, Townsend N, Gale CP, et al. European Society of Cardiology: cardiovascular disease statistics 2019. Eur Heart J 2020;41:12-85.

3 Roth GA, Johnson C, Abajobir A, et al. Global, regional, and national burden of cardiovascular diseases for 10 causes, 1990 to 2015. J Am Coll Cardiol 2017;70:1-25

4 Reiner Željko. Hypertriglyceridaemia and risk of coronary artery disease. Nat Rev Cardiol 2017;14:401-11.

5 Arnett DK, Blumenthal RS, Albert MA, et al. 2019 ACC/AHA guideline on the primary prevention of cardiovascular disease: Executive summary: a report of the American College of Cardiology/American heart association Task force on clinical practice guidelines. Circulation 2019;140:e563-95.

6 Arsenault BJ, Boekholdt SM, Kastelein JJP. Lipid parameters for measuring risk of cardiovascular disease. Nat Rev Cardiol 2011;8:197-206.

7 Mach F, Baigent C, Catapano AL, et al. 2019 ESC/EAS guidelines for the management of dyslipidaemias: lipid modification to reduce cardiovascular risk. Eur Heart J 2020;41:111-88.

8 Chapman MJ, Ginsberg HN, Amarenco P, et al. Triglyceride-rich lipoproteins and high-density lipoprotein cholesterol in patients at high risk of cardiovascular disease: evidence and guidance for management. Eur Heart $\mathrm{J}$ 2011;32:1345-61.

9 Ouimet M, Barrett TJ, Fisher EA. HDL and reverse cholesterol transport. Circ Res 2019;124:1505-18.

10 Rosenson RS, Brewer HB, Ansell BJ, et al. Dysfunctional HDL and atherosclerotic cardiovascular disease. Nat Rev Cardiol 2016;13:48-60.

11 Ferrari R, Aguiar C, Alegria E, et al. Current practice in identifying and treating cardiovascular risk, with a focus on residual risk associated with atherogenic dyslipidaemia. Eur Heart J Supp/ 2016;18:C2-12.

12 Catapano AL, Graham I, De Backer G, et al. 2016 ESC/EAS guidelines for the management of Dyslipidaemias. Eur Heart J 2016:37:2999-3058.

13 Langlois MR, Chapman MJ, Cobbaert C, et al. Quantifying atherogenic lipoproteins: current and future challenges in the era of personalized medicine and very low concentrations of LDL cholesterol. A consensus statement from EAS and EFLM. Clin Chem 2018;64:1006-33.

14 Barrett TJ, Distel E, Murphy AJ, et al. Apolipoprotein Al) promotes atherosclerosis regression in diabetic mice by suppressing myelopoiesis and plaque inflammation. Circulation 2019;140:1170-84.

15 Henson D, Tahhan AS, Nardo D, et al. Association between ApoA-I (apolipoprotein A-I) immune complexes and adverse cardiovascular EventsBrief report. Arterioscler Thromb Vasc Biol 2019;39:1884-92.

16 Karthikeyan G, Teo KK, Islam S, et al. Lipid profile, plasma apolipoproteins, and risk of a first myocardial infarction among Asians: an analysis from the INTERHEART study. J Am Coll Cardiol 2009;53:244-53.

17 Patel JV, Abraheem A, Creamer J, et al. Apolipoproteins in the discrimination of atherosclerotic burden and cardiac function in patients with stable coronary artery disease. Eur J Heart Fail 2010;12:254-9.

18 Thygesen K, Alpert JS, White HD, et al. Universal definition of myocardial infarction. Eur Heart J 2007;28:2525-38.

19 Faul F, Erdfelder E, Buchner A, et al. Statistical power analyses using $G^{*}$ Power 3.1: tests for correlation and regression analyses. Behav Res Methods 2009:41:1149-60.

20 Prakash AM, Sun Y, Chiaramida SA, et al. Quantitative assessment of pericardial effusion volume by two-dimensional echocardiography. J Am Soc Echocardiogr 2003;16:147-53.
21 Fox KAA, Dabbous OH, Goldberg RJ, et al. Prediction of risk of death and myocardial infarction in the six months after presentation with acute coronary syndrome: prospective multinational observational study (GRACE). BMJ 2006;333:1091.

22 Winter M-P, Wiesbauer F, Blessberger $\mathrm{H}$, et al. Lipid profile and longterm outcome in premature myocardial infarction. Eur J Clin Invest 2018:48:e13008.

23 Welsh C, Celis-Morales CA, Brown R, et al. Comparison of conventional lipoprotein tests and apolipoproteins in the prediction of cardiovascular disease. Circulation 2019;140:542-52.

24 Holme I, Strandberg TE, Faergeman 0, et al. Congestive heart failure is associated with lipoprotein components in statin-treated patients with coronary heart disease insights from the incremental decrease in end points through aggressive lipid lowering trial (ideal). Atherosclerosis 2009;205:522-7.

25 Wedel H, McMurray JJV, Lindberg M, et al. Predictors of fatal and non-fatal outcomes in the controlled rosuvastatin multinational trial in heart failure (CORONA): incremental value of apolipoprotein A-1, high-sensitivity C-reactive peptide and N-terminal pro B-type natriuretic peptide. Eur J Heart Fail 2009;11:281-91.

26 Prats-Uribe A, Sayols-Baixeras S, Fernández-Sanlés A, et al. High-density lipoprotein characteristics and coronary artery disease: a Mendelian randomization study. Metabolism 2020;112:154351.

27 Meikle PJ, Formosa MF, Mellett NA, et al. HDL phospholipids, but not cholesterol distinguish acute coronary syndrome from stable coronary artery disease. J Am Heart Assoc 2019;8:e011792.

28 Capodanno D, Mehran R, Gibson CM, et al. CSL112, a reconstituted, infusible, plasma-derived apolipoprotein A-l: safety and tolerability profiles and implications for management in patients with myocardial infarction. Expert Opin Investig Drugs 2018;27:997-1005.

29 Gibson CM, Korjian S, Tricoci P, et al. Rationale and design of Apo-I event reduction in ischemic syndromes I (AEGIS-I): a phase $2 \mathrm{~B}$, randomized, placebo-controlled, dose-ranging trial to investigate the safety and tolerability of CSL112, a reconstituted, infusible, human apoA-I, after acute myocardial infarction. Am Heart J 2016;180:22-8.

30 Michael Gibson C, Korjian S, Tricoci P, et al. Safety and tolerability of CSL112, a reconstituted, Infusible, plasma-derived apolipoprotein A-I, after acute myocardial infarction: the AEGIS-I trial (ApoA-I event reducing in ischemic syndromes I). Circulation 2016;134:1918-30.

31 Boekholdt SM, Arsenault BJ, Hovingh GK, et al. Levels and changes of $\mathrm{HDL}$ cholesterol and apolipoprotein A-I in relation to risk of cardiovascular events among statin-treated patients: a meta-analysis. Circulation 2013;128:1504-12

32 Mora S, Glynn RJ, Ridker PM. High-density lipoprotein cholesterol, size, particle number, and residual vascular risk after potent statin therapy. Circulation 2013;128:1189-97.

33 Duparc T, Ruidavets J-B, Genoux A, et al. Serum level of HDL particles are independently associated with long-term prognosis in patients with coronary artery disease: the genes study. Sci Rep 2020;10:8138.

34 Sandhu PK, Musaad SMA, Remaley AT, et al. Lipoprotein biomarkers and risk of cardiovascular disease: a laboratory medicine best practices (LMBP) systematic review. J App/ Lab Med 2016;1:214-29.

35 Javaheri A, Rader DJ. Apolipoprotein A-I and cholesterol efflux: the good, the bad, and the modified. Circ Res 2014;114:1681-3.

36 Rosenson RS, Brewer HB, Rader DJ. Lipoproteins as biomarkers and therapeutic targets in the setting of acute coronary syndrome. Circ Res 2014;114:1880-9. 